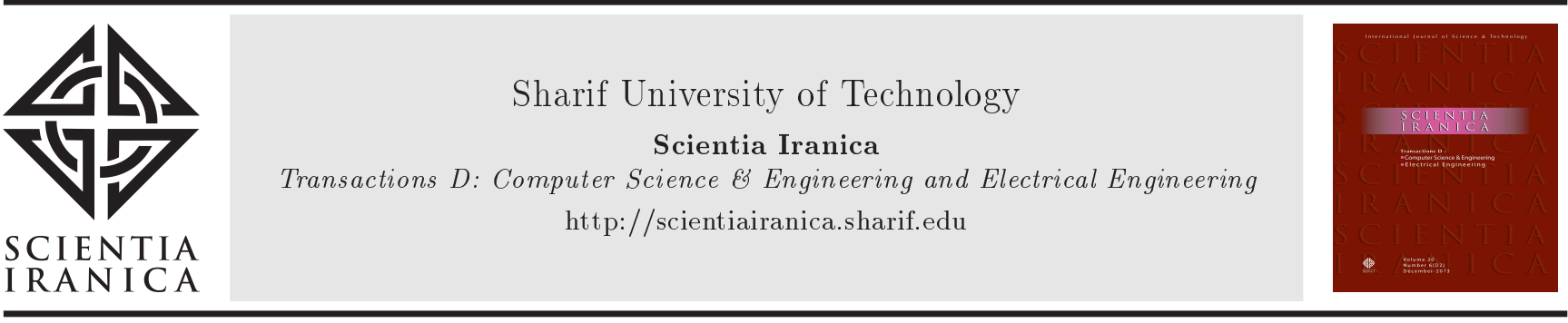

\title{
Shannon entropy and tracking dynamic systems over noisy channels
}

\author{
A. Farhadi* \\ Department of Electrical Engineering at Sharif University of Technology, Tehran, Iran.
}

Received 31 October 2015; received in revised form 30 November 2016; accepted 31 December 2016

\section{KEYWORDS}

Estimation;

Networked control system;

Shannon entropy.

\begin{abstract}
This paper is concerned with the estimation of state trajectory of linear discrete time dynamic systems subject to parametric uncertainty over the compound erasure channel that uses feedback channel intermittently. For this combination of system and channel, using the data processing inequality and a robust version of the Shannon lower bound, a necessary condition for channel capacity in estimation of state trajectory at the receiver, giving almost sure asymptotically zero estimation error, is presented. Then, an estimation technique over the compound erasure channel that includes an encoder, decoder, and a sufficient condition under which the estimation error at the receiver is asymptotically zero almost surely is presented. This leads to the conclusion that over the compound erasure channel, a condition for Shannon capacity in terms of its rate of expansion is necessary and sufficient in estimation with uniform almost sure asymptotically zero estimation error. The satisfactory performance of the proposed technique is illustrated using simulation.
\end{abstract}

(C) 2018 Sharif University of Technology. All rights reserved.

\section{Introduction}

\subsection{Motivation and background}

One of the issues that has begun to emerge in a number of applications, such as networked control systems [1$22]$, is how to estimate the state trajectory of a dynamic system over a communication channel subject to imperfections (e.g., noise and limited capacity). In these applications, estimation means how to transmit information about the state trajectory of a dynamic system and reconstruct it reliably in real time at the receiver. In these applications, it is essential to find methodologies for designing proper estimator over, for example, data links subject to data dropout and limited capacity.

In this paper, we consider the problem of estimating the state trajectory of a linear time-invariant

\footnotetext{
*. E-mail address: afarhadi@sharif.edu
}

dynamic system subject to parametric uncertainty over the limited-capacity compound erasure channel (i.e., the packet erasure channel with unknown erasure probability) that uses feedback channel with duty cycle, $\beta \in(0,1]$; where $\beta$ is a rational number as shown in Figure 1. $\beta=0$ means non-availability of feedback channel while $\beta=1$ means full-time availability of feedback channel. In some emerging applications, such as tele-operation of micro autonomous Unmanned Aerial Vehicles (UAVs) and Autonomous Underwater Vehicles (AUVs), it is necessary to transmit the observation signal of the dynamic system to remote base station where the controller is located over a limitedcapacity communication channel subject to imperfections. Thus, in these applications, it is necessary to estimate the state trajectory of the dynamic system using the transmitted observation signal over communication channel to produce the proper control commands. The block diagram of Figure 1 presents the block diagram of this estimation problem that occurs in tele-operation of micro UAVs and AUVs. 


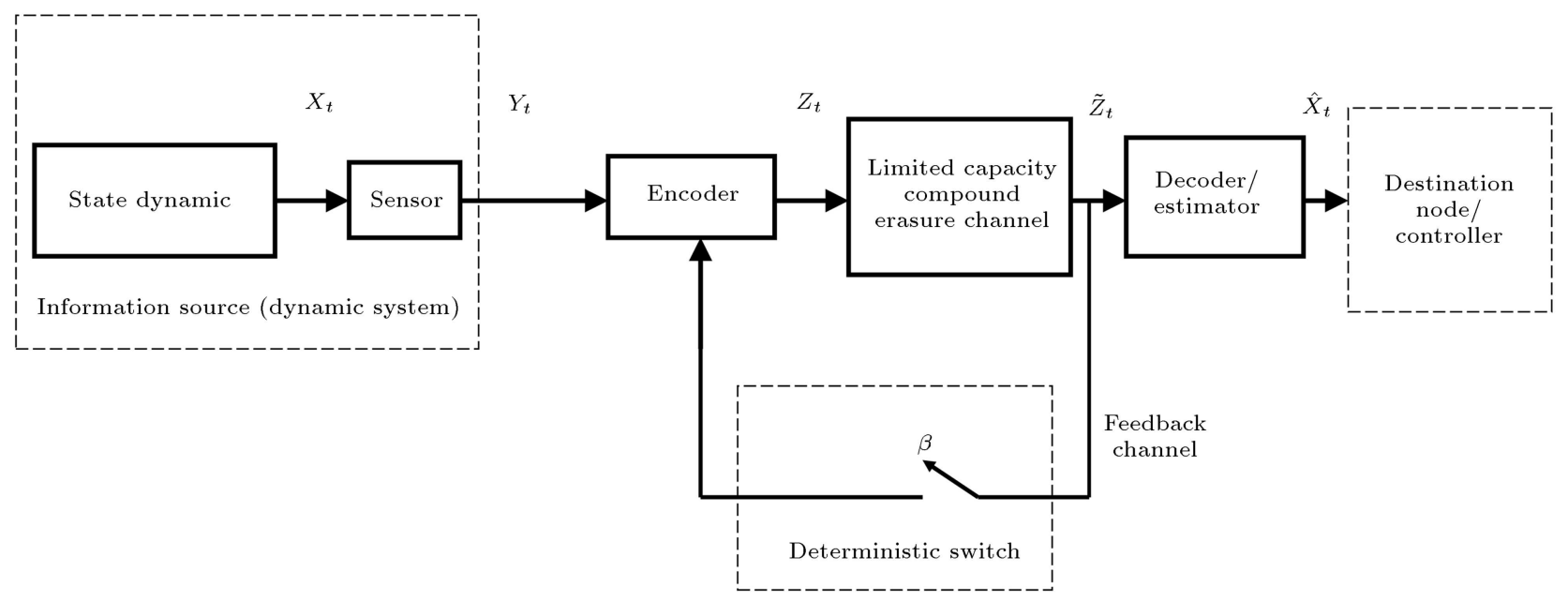

Figure 1. Communication System (CS).

Although it is known in the context of information theory that feedback channel does not increase the capacity of Discrete Memoryless Channels (DMCs), it significantly simplifies the design of encoder and decoder [23], compensating for the effects of channel imperfections and reliably estimating the state of the system in control over communication problems. The simplicity of the design of encoder and decoder is a required property in control over communication as complicated design results in significant time latency between making measurements from dynamic system and applying the corresponding control commands, which obviously damages the performance. This motivated us to use feedback channel in the block diagram of Figure 1. Nevertheless, the full-time availability of feedback channel (i.e., $\beta=1$ ) in the block diagram of Figure 1 requires the feedback channel signal to be transmitted with high power full-time. This results in significant power consumption at receiver in order to transmit noiseless feedback channel fulltime. Therefore, it is more desirable to use noiseless feedback channel that is only available intermittently (i.e., $\beta \in(0,1))$ to avoid exhausting receiver power supply.

In the block diagram of Figure 1, the communication channel is a limited-capacity compound erasure channel. It is a packet erasure channel [18] with unknown erasure probability. The packet erasure channel is an abstract model for the commonly used information technologies, such as the Internet, WiFi, and mobile communication; accordingly, we consider it in this paper.

As linear dynamic systems are an important class of systems, in the block diagram of Figure 1, we are concerned with the class of linear time-invariant dynamic systems. Having chosen a suitable dynamic model for the system, the defining parameters of the system are usually estimated from a sample of experimental data. However, there is always uncertainty associated with any estimation. This results in parametric uncertainty in many models. Therefore, systems subject to parametric uncertainty form a large and important class of dynamic systems and have been considered in many studies, such as [24-27]. Hence, in this paper, we are concerned with the state estimation of linear time-invariant dynamic systems subject to parametric uncertainty over the compound erasure channel.

The problem of almost sure estimation of linear time-invariant dynamic systems over the packet erasure channel that uses feedback channel full-time has been addressed in the literature (e.g., [11,18]). In [18], it was shown that the eigenvalues rate condition described by the Shannon capacity was tight. That is, the following condition:

$$
\mathcal{C} \geq \sum_{\left\{i:\left|\lambda_{i}(A)\right| \geq 1\right\}} \log \left|\lambda_{i}(A)\right|,
$$

where $\mathcal{C}$ denotes the capacity of the DMCs and $\lambda_{i}(A)$ denotes the eigenvalues of linear time-invariant noiseless dynamic system, is the necessary and sufficient condition for an estimation over DMCs with almost sure asymptotically zero estimation error. Linear time-invariant dynamic systems subject to uniformly bounded exogenous disturbances over the DMCs (with and without feedback channel) have also been considered in the literature, and it is shown that the following condition:

$$
\mathcal{C}_{0} \geq \sum_{\left\{i:\left|\lambda_{i}(A)\right| \geq 1\right\}} \log \left|\lambda_{i}(A)\right|,
$$

where $\mathcal{C}_{0}$ denotes the Shannon zero error capacity [23], is the necessary and sufficient condition for estimation over DMCs (with and without feedback channel) giving almost sure asymptotically bounded estimation error [22] (i.e., $\lim \sup _{t \rightarrow \infty}\left\|X_{t}-\hat{X}_{t}\right\|<\infty$, where 
$X_{t}$ is the state of the system and $\hat{X}_{t}$ is its estimate). As the Shannon zero error capacity of noisy DMCs with and without feedback channel is zero, this result indicates that we cannot estimate the states of linear time-invariant dynamic systems subject to uniformly bounded exogenous disturbances over noisy communication channels. The problem of almost sure bounded stability of controlled nonlinear Lipschitz systems over the digital noiseless channel was addressed in [17], in which a sufficient condition relating transmission rate to Lipschitz coefficient was presented for almost sure asymptotic bounded stability. Note that the problem addressed in this paper is quite different as we consider different dynamic system, different communication channel, and different objective. In this paper, we present the necessary and sufficient condition for uniform almost sure asymptotic estimation of linear time-invariant dynamic systems over the limitedcapacity compound erasure channel. The problem of optimal reference tracking of linear time-invariant dynamic systems over Additive White Gaussian Noise (AWGN) channel was addressed in [19]. Also, the problem of optimal reference tracking of linear timeinvariant dynamic systems over the packet erasure channel with known erasure probability was addressed in [20]. Moreover, the problem of optimal reference tracking of linear time-invariant systems over AWGN channel in feedback path or forward path was addressed in [21]. Note that in $[19,20]$, the objective is to make the system output follow a desired reference signal, while in this paper, the objective is to estimate the state trajectory of the system at the end of communication, as shown in Figure 1.

\subsection{Paper contributions}

The main novelty of this paper is in the necessary and sufficient condition for Uniform Almost Sure Asymptotic Estimation (UASAE) over the limited capacity compound erasure channel.

For the block diagram of Figure 1, we present necessary and sufficient conditions that provide UASAE when feedback channel is not necessarily available fulltime. Using an information theoretic approach, we derive a necessary condition for this type of estimation over the compound erasure channel. This necessary condition is given in terms of the Shannon capacity and the Shannon lower bound, which are related to the rate of expansion of the Shannon entropy of the dynamic system. This leads to the eigenvalues rate condition described by the Shannon capacity. In the absence of uncertainty in the dynamic system, we are also able to present a sufficient condition for which UASAE holds over the compound erasure channel. The sufficient condition is also given in terms of the rate of expansion of the Shannon entropy of the dynamic system. Hence, this paper extends the previous results (e.g. [18]) to cases in which both the dynamic system and communication channel are subject to uncertainty and feedback channel is not necessarily available full-time. It also complements the previous results (e.g. [22]) by considering parametric uncertainty instead of uniformly bounded disturbances and showing that in the presence of uncertainty in the communication channel, the eigenvalues rate condition described by the Shannon capacity is a tight bound for UASAE.

\subsection{Paper organization}

The paper is organized as follows. Section 2 is devoted to problem formulation. In this section, we describe the compound erasure channel and the notion of uniform almost sure asymptotic estimation. In Section 3, we first describe the notions of Shannon capacity, rate distortion, and Shannon entropy. Then, a necessary condition for UASAE is presented. In Section 4, a sufficient condition for this type of estimation is given. Finally, the paper is concluded in Section 5. Proofs are given in the Appendix.

\section{Problem formulation}

This paper is concerned with the communication system (CS) shown in Figure 1, which is defined in a complete probability space $(\Omega, \mathcal{F}(\Omega), P)$ with filtration $\left\{\mathcal{F}_{t}\right\}_{t>0}$. Here, $X_{t}, Y_{t}, Z_{t}, \tilde{Z}_{t}$, and $\hat{X}_{t}$ are random vectors denoting the state of the dynamic system, observation, channel input, channel output, and the state estimate, respectively, at time $t \in \mathbf{N}_{+} \equiv\{0,1,2, \cdots\}$. Note that $X_{t} \in \mathbf{R}^{q}, Y_{t} \in \mathbf{R}^{d}$, and $\hat{X}_{t} \in \mathbf{R}^{q}$. In the CS shown in Figure 1, we can use feedback channel with duty cycle, $\beta \in(0,1]$, where $\beta$ is a rational number. $\beta=0$ corresponds to the case of non-availability of feedback channel while $\beta=1$ corresponds to its fulltime availability. This is shown by a switch with a known policy.

Throughout the paper, we adopt the following notation. Random Vectors (R.V.s) are denoted by capital letters, while a realization of a R.V. is denoted by a lower-case letter. Sequences of R.V.s are denoted by $Y_{0}^{T} \equiv\left(Y_{0}, Y_{1}, \cdots, Y_{T}\right)$. We denote by $A^{\prime}$ the transpose of $A$, where $A$ is either a matrix or a vector, and by $A^{-1}$ the inverse of a square invertible matrix $A$. We denote by $\|\cdot\|$ the Euclidean norm on the vector space, $\mathbf{R}^{q}$; by $|x|$ the absolute value of a scalar, $x \in \mathbf{R}$; and by $M(q \times o)$ the space of all matrices, $A \in \mathbf{R}^{q \times o}$. The space $M(q \times o)$ is endowed with the spectral norm, $\|A\| \equiv \sqrt{\lambda_{\max }\left(A^{\prime} A\right)}$, where $\lambda_{\max }\left(A^{\prime} A\right)$ is the largest eigenvalue of the matrix $A^{\prime} A$. We denote by $\mathcal{B}(\mathcal{X})$ the Borel $\sigma$-algebra generated by the open subsets of the non-empty (arbitrary) set $\mathcal{X}$ and by $(\mathcal{X}, \mathcal{B}(\mathcal{X}))$ the Borel measurable space. We also denote by $\mathcal{M}_{1}(\mathcal{X})$ the space of probability measures defined in the measurable 
space $(\mathcal{X}, \mathcal{B}(\mathcal{X}))$. Given a pair of measurable spaces $(\bar{A}, \mathcal{B}(\bar{A}))$ and $(\hat{A}, \mathcal{B}(\hat{A}))$, a mapping, $Q: \mathcal{B}(\hat{A}) \times \bar{A} \rightarrow$ $[0,1]$, is called a stochastic kernel if it satisfies the following two properties:

i) For every $x \in \bar{A}$, the set function $Q(\cdot \mid x)$ is a probability measure on $\hat{A}$;

ii) For every $F \in \mathcal{B}(\hat{A})$, the function $Q(F \mid \cdot)$ is $\bar{A}$ measurable.

Time ordered products are denoted by $\prod_{t \geq k \geq 0} a_{k} \equiv$ $a_{t} \cdot a_{t-1} \cdots a_{0}$.

The different blocks of Figure 1 representing the CS are described below:

- Information source: The information source is described by a dynamic system subject to parametric uncertainty.

In this paper, we are concerned with an information source described by the following uncertain linear discrete time dynamic system:

$$
\begin{aligned}
& X_{t+1}=\left(A+\Gamma_{t}\right) X_{t}, \quad X_{0}=x_{o}, \quad A \neq 0, \\
& \quad \Gamma_{t} \in B_{\ell}(M(q \times q)), \\
& Y_{t}=C X_{t},
\end{aligned}
$$

where $X_{t} \in \mathbf{R}^{q}$ is the state of the system at time $t \in \mathbf{N}_{+} ; A \in M(q \times q)$ is the nominal (known) system matrix; initial condition, $X_{0}$, has a known density denoted by $p_{0} ; Y_{t} \in \mathbf{R}^{d}$ is the observation (the source output); and $C \in M(d \times q)$. The unknown matrix $\Gamma_{t} \in M(q \times q)$ represents the uncertainty in the system parameters. At any time $t \in \mathbf{N}_{+}$, it is a measurable function:

$$
\begin{aligned}
& \Gamma_{t}:(\Omega, \mathcal{F}(\Omega)) \rightarrow B_{\ell}(M(q \times q)) \\
& \quad \equiv\{\Gamma \in M(q \times q):\|\Gamma\| \leq \ell\}
\end{aligned}
$$

where $\ell$ is a known non-negative scalar representing the radius of uncertainty.

Let $P_{t}^{x}, t \in \mathbf{N}_{+}$denote the probability measure associated with the state variable, $X_{t} \in \mathbf{R}^{q}$, i.e., $P_{t}^{x}(B) \equiv P\left(X_{t} \in B\right), B \in \mathcal{B}\left(\mathbf{R}^{q}\right)$. We assume that $P_{t}^{x}$ admits of a density function $p_{t}$, i.e., $P_{t}^{x}(B)=$ $\int_{B} p_{t}(x) d x, \forall B \in \mathcal{B}\left(\mathbf{R}^{q}\right)$. Since System (1) is uncertain, the information source is also uncertain, in which the uncertainty in source is characterized by a family of probability measures, $\mathcal{M}_{S, t}\left(\subset \mathcal{M}_{1}\left(\mathbf{R}^{q}\right)\right)$, induced by R.V. $X_{t}=\left(\prod_{t-1>k>0}\left(A+\Gamma_{k}\right)\right) X_{0}$, where $\Gamma_{k}$ takes values from the set $\bar{B}_{\ell}(M(q \times q))$. In other words, the source is represented by the family of probability measures, $P_{t}^{x} \in \mathcal{M}_{S, t}$. Note that the case $\ell=0$ corresponds to a known system, which is the nominal system;
- Communication channel: From the bit-wise perspective, the channel input is ' 0 ,' ' 1 ,' and 'idle' and the channel output is ' 0 ,' ' 1 ,' and 'idle'. That is, when the channel is not in use, it is in 'idle' mode. But, from the packet-wise perspective, in this paper, we are concerned with the discrete memoryless limited-capacity compound erasure channel. It is a packet erasure channel [23] with the channel input alphabet $\mathbf{Z}=\{0,1\}^{\mathcal{R}}$ (where $\mathcal{R} \in\{1,2,3, \cdots\}$ is the length of transmitted packet in a channel use) and the output alphabet $\tilde{\mathbf{Z}}=\mathbf{Z} \bigcup\{e\}$ (where $e$ stands for the erasure symbol). The erasure probability $\theta$ is unknown to both transmitter and receiver. However, the unknown erasure probability $\theta$ belongs to a known set $\Theta$, which is a compact (and proper) subset of $[0,1)$;

- Encoder: We introduce three classes of encoders, namely, Class A, Class B, and Class C. Let $E \in$ $\mathcal{B}(\mathbf{Z})$ and $\bar{z}_{0}^{t-1}=\left(r_{0} \tilde{z}_{0}, r_{1} \tilde{z}_{1}, \cdots, r_{t-1} \tilde{z}_{t-1}\right)$, where $r_{j}=0$ corresponds to the case of inactive feedback channel at time instant $j$ and $r_{j}=1$ corresponds to the feedback channel being active. At any time $t \in \mathbf{N}_{+}$, Class A, Class B, and Class $\mathrm{C}$ encoders are modeled by stochastic kernels $Q_{t}^{A}\left(E \mid y_{0}^{t}, z_{0}^{t-1}\right)$, $Q_{t}^{B}\left(E \mid y_{0}^{t}, z_{0}^{t-1}, \tilde{z}_{0}^{t-1}\right)$, and $Q_{t}^{C}\left(E \mid y_{0}^{t}, z_{0}^{t-1}, \bar{z}_{0}^{t-1}\right)$, respectively. Note that the Class A encoder does not use the channel outputs, Class B encoder can use all the channel outputs up to time $t-1$ via feedback channel, and Class C encoders can only use (via feedback) some of the channel outputs;

- Decoder/estimator: At any time $t \in \mathbf{N}_{+}$the decoder is a mapping of the channel outputs $\tilde{Z}_{0}^{t}$ onto the state estimate $\hat{X}_{t} \in \mathbf{R}^{q}$. It is described by a stochastic kernel $Q_{t}^{D}\left(E \mid \tilde{z}_{0}^{t}\right), E \in \mathcal{B}\left(\mathbf{R}^{q}\right)$. Note that as the channel output is ternary, the decoder can identify the length of transmitted packet;

- Deterministic switch: In many practical applications, providing a noiseless feedback acknowledgment from receiver to transmitter in each time step is difficult and/or expensive. Therefore, in this paper, we use feedback channel with duty cycle, $\beta \in(0,1]$, where $\beta=0$ corresponds to the inactive state of the feedback channel and $\beta=1$ corresponds to the active state (i.e., available all the time). This is shown by a switch with a known switching policy in the CS shown in Figure 1.

In many applications, a tracker of a signal process, giving almost sure zero estimation error, is desirable as it results in almost sure stability of the controlled system. Therefore, in this paper, we are concerned with this type of estimation. The objective is to find necessary and sufficient conditions for the Shannon capacity in which UASAE, as defined below, holds. 
Definition 2.1. $\quad$ Consider the CS shown in Figure 1 described by the uncertain System (1) and the compound erasure channel. For System (1), UASAE holds if there exist an encoder and a decoder such that for any $\epsilon>0$, there exists a finite time $T(\epsilon) \geq 0$, such that:

$$
P\left(\sup _{t \geq T(\epsilon)}\left\|X_{t}-\hat{X}_{t}\right\|>\epsilon\right) \leq \epsilon
$$

$\forall \Gamma_{k} \in B_{\ell}(M(q \times q))(k \leq t-1)$ and $\forall \theta \in \Theta$.

Throughout the paper, it is assumed that the erasure probability $\theta$ and the distribution of $\Gamma_{t}$ are not known to transmitter and receiver. But, the set $\Theta$, the non-negative scalar representing the radius of uncertainty, $\ell$, and $\beta$ are known a priori.

\section{Necessary condition}

In this section, a necessary condition in which UASAE holds is derived for the CS shown in Figure 1. This condition is obtained by establishing a relationship between the Shannon capacity, robust rate distortion, and a variant of the Shannon lower bound. These are information theoretic measures, which we will recall in this paper.

Consider the compound erasure channel, as described earlier, and let $Z_{0}^{t}$ and $\tilde{Z}_{0}^{t}$ be sequences of the channel input and output symbols, respectively. Let $\mathcal{Z}_{0, t} \equiv \prod_{k=0}^{t} \mathbf{Z}$ denote the space which contains $Z_{0}^{t}$ (i.e., $\left.Z_{0}^{t} \in \mathcal{Z}_{0, t}\right)$. For any given erasure probability $\theta \in \Theta$, let $Q_{0, t}^{\theta}(d \tilde{z} \mid z)$ denote the stochastic kernel corresponding to $\tilde{Z}_{0}^{t}$ and $Z_{0}^{t}$. Further, let $P_{0, t}^{z}(B) \equiv P\left(Z_{0}^{t} \in B\right), B \in$ $\mathcal{B}\left(\mathcal{Z}_{0, t}\right)$ denote the probability measure corresponding to the sequence $Z_{0}^{t}$. The Shannon capacity of the above channel, which is the maximum rate in bits per channel use at which information can be sent with arbitrarily low probability of error, is defined as follows.

Definition 3.1 (The Shannon capacity of the compound erasure channel) [28]. Consider the compound erasure channel, as described above. The capacity of this channel for $t+1$ channel uses is defined by $\mathcal{C}_{t} \equiv \sup _{P_{0, t}^{z} \in \mathcal{M}_{1}\left(\mathcal{Z}_{0, t}\right)} \inf _{\theta \in \Theta} I^{\theta}\left(Z_{0}^{t}, \tilde{Z}_{0}^{t}\right)$ where:

$$
\begin{aligned}
I^{\theta}\left(Z_{0}^{t}, \tilde{Z}_{0}^{t}\right) \equiv & \iint \log \left(\frac{Q_{0, t}^{\theta}(d \tilde{z} \mid z)}{\int Q_{0, t}^{\theta}(d \tilde{z} \mid z) P_{0, t}^{z}(d z)}\right) \\
& Q_{0, t}^{\theta}(d \tilde{z} \mid z) P_{0, t}^{z}(d z)
\end{aligned}
$$

denotes the mutual information between sequences $Z_{0}^{t}$ and $\tilde{Z}_{0}^{t}$ (and the superscript $\theta$ emphasizes the dependency of the mutual information on parameter $\theta$ ). Then, the Shannon capacity in bits per channel use is given by $\mathcal{C} \equiv \lim _{t \rightarrow \infty} \frac{1}{t+1} \mathcal{C}_{t}$.
For the compound erasure channel, the capacity for achieving input probability mass function is the same for all the channels in the family $\theta \in \Theta$. Therefore, for these channels, we have [29]:

$$
\begin{aligned}
\mathcal{C}_{t} & \equiv \sup _{P_{0, t}^{z} \in \mathcal{M}_{1}\left(\mathcal{Z}_{0, t}\right)} \inf _{\theta \in \Theta} I^{\theta}\left(Z_{0}^{t}, \tilde{Z}_{0}^{t}\right) \\
& =\inf _{\theta \in \Theta} \sup _{P_{0, t}^{z} \in \mathcal{M}_{1}\left(\mathcal{Z}_{0, t}\right)} I^{\theta}\left(Z_{0}^{t}, \tilde{Z}_{0}^{t}\right) .
\end{aligned}
$$

It is shown in [30] that the feedback capacity of the memoryless compound channel is given by:

$$
\mathcal{C}=\lim _{t \rightarrow \infty} \frac{1}{t+1} \mathcal{C}_{t}
$$

where:

$$
\mathcal{C}_{t}=\inf _{\theta \in \Theta} \sup _{P_{0, t}^{z} \in \mathcal{M}_{1}\left(\mathcal{Z}_{0, t}\right)} I^{\theta}\left(Z_{0}^{t}, \tilde{Z}_{0}^{t}\right) .
$$

Hence, it follows from Eq. (2) that feedback does not increase the capacity of the compound erasure channel. However, as shown in [23], it can significantly help in simplifying coding scheme. Note that for a compound erasure channel with the channel input alphabet $\mathbf{Z}=$ $\{0,1\}^{\mathcal{R}}$ (where $\mathcal{R}$ is the length of transmitted packet in each channel use), output alphabet $\tilde{\mathbf{Z}}=\mathbf{Z} \cup\{e\}$, and unknown erasure probability $\theta \in \Theta$, it is verified that $\mathcal{C}_{t}=\inf _{\theta \in \Theta}(1-\theta)(t+1) \mathcal{R}$ and therefore, $\mathcal{C}=$ $\inf _{\theta \in \Theta}(1-\theta) \mathcal{R}$.

Next, we recall the definition of robust rate distortion and then, we establish a relationship between the Shannon capacity and the robust rate distortion for reliable data reconstruction. Let $X_{t} \in \mathbf{R}^{q}$ denote the source message with distribution $P_{t}^{x}$ and $\hat{X}_{t} \in \mathbf{R}^{q}$ the corresponding reconstruction. Suppose that the source is uncertain in the sense that its probability measure is unknown, but the set $\mathcal{M}_{S, t} \subset \mathcal{M}_{1}\left(\mathbf{R}^{q}\right)$ to which it belongs is known. That is, $P_{t}^{x} \in \mathcal{M}_{S, t} \subset \mathcal{M}_{1}\left(\mathbf{R}^{q}\right)$. Let $D \geq 0$ denote the distortion level and let:

$$
\begin{aligned}
\mathcal{M}_{D}\left(P_{t}^{x}\right) \equiv & \left\{Q_{t}: \int_{\mathbf{R}^{q}} \int_{\mathbf{R}^{q}} \rho(x-\hat{x}) Q_{t}(d \hat{x} \mid x)\right. \\
& \left.\times P_{t}^{x}(d x) \leq D\right\},
\end{aligned}
$$

represent the set of stochastic kernels satisfying the distortion constraint where $\rho(x-\hat{x})$ is the difference distortion measure. For example, the distortion measure $\rho$ can be chosen either as $r$-moment measure (i.e., $\rho_{r}(x-\hat{x}) \equiv\|x-\hat{x}\|^{r}, r>0$ or as indicator measure, i.e.,

$$
\rho_{\epsilon}(x-\hat{x}) \equiv\left\{\begin{array}{ll}
0 & \text { if }\|x-\hat{x}\| \leq \epsilon \\
1 & \text { if }\|x-\hat{x}\|>\epsilon
\end{array} \quad(\epsilon>0)\right.
$$

Then, the robust rate distortion for the uncertain source is defined as follows. 
Definition 3.2 (The robust rate distortion) [31]. Consider the information source as described above. The robust rate distortion corresponding to the family $\mathcal{M}_{S, t}$ is given by:

$$
R_{t}(D) \equiv \inf _{Q_{t} \in \mathcal{M}_{D}\left(P_{t}^{x}\right)} \sup _{P_{t}^{x} \in \mathcal{M}_{S, t}} I\left(X_{t}, \hat{X}_{t}\right) .
$$

As shown in [31], when $\mathcal{M}_{S, t}$ is a compact set, inf sup in Eq. (4) can be exchanged with sup inf. Furthermore, when the messages are produced according to an i.i.d. distribution, the rate distortion $R_{t}(D)$, as defined above, has an operational meaning and it represents the minimum rate for which uniform reliable data reconstruction up to the distortion level $D$ holds.

For most of distortion measures and source distributions, finding an explicit analytical expression for the rate distortion as a function of $t$ and $D$ is difficult. Therefore, approximating the rate distortion function by a lower bound, which can be easily computed, is useful. In the following lemma, we present a lower bound for the (robust) rate distortion in terms of the (robust) entropy of the source message. We use this lemma to present a necessary condition for UASAE.

Toward this goal, consider the uncertain information source, as described above, and suppose that $P_{t}^{x}$ admits of a density function $p_{t}$. Denote the Shannon (differential) entropy associated with the density function $p_{t}$ by $H\left(p_{t}\right)$, which is given by [23]:

$$
H\left(p_{t}\right) \equiv-\int_{\mathbf{R}^{q}} p_{t}(x) \log \left(p_{t}(x)\right) d x .
$$

Also, let $R_{S, t}(D)$ be the corresponding Shannon lower bound given by [32]:

$$
R_{S, t}(D) \equiv H\left(p_{t}\right)-\max _{h \in G_{D}} H(h),
$$

where:

$$
\begin{aligned}
G_{D} & \equiv\left\{h: \mathbf{R}^{q} \rightarrow[0, \infty): \int_{\mathbf{R}^{q}} h(\xi) d \xi=1, \int_{\mathbf{R}^{q}} \rho(\xi) h(\xi) d \xi\right. \\
& \leq D\}
\end{aligned}
$$

and $H(h)$ is the Shannon entropy associated with the density $h$. Note that when:

$$
\int_{\mathbf{R}^{q}} e^{s \rho(\xi)} d \xi<\infty \quad \text { for all } \quad s<0
$$

the density $h^{*} \in G_{D}$ that maximizes $H(h)$ is given by:

$$
h^{*}(\xi)=\frac{e^{s^{*} \rho(\xi)}}{\int_{\mathbf{R}^{q}} e^{s^{*} \rho(\xi)} d \xi},
$$

for some $s^{*}<0$ satisfying $\int_{\mathbf{R}^{q}} \rho(\xi) h^{*}(\xi) d \xi=D$.

Then, a relationship between the robust rate distortion and the Shannon lower bound for the uncertain information source is given by the following result:
Lemma 3.3 (The Shannon lower bound). Consider the information source, as described above. Suppose that $P_{t}^{x}$ admits of a density function $p_{t}$. Then:

(i) For the case of single source (i.e., $\mathcal{M}_{S, t}=\left\{P_{t}^{x}\right\}$ ), a lower bound for the rate distortion function $R_{t}(D)$ is given by $R_{t}(D) \geq R_{S, t}(D) \equiv H\left(p_{t}\right)-$ $\max _{h \in G_{D}} H(h)$;

(ii) For the case of uncertain source, a lower bound for the robust rate distortion is given by $R_{t}(D) \geq$ $\sup _{P_{t}^{x} \in \mathcal{M}_{S, t}} H\left(p_{t}\right)-\max _{h \in G_{D}} H(h)$.

Proof. See Appendix.

Consider the CS shown in Figure 1, with the corresponding mathematical model as described earlier. At time $t$, the state of the system $X_{t}$ is observed and the observation is encoded and transmitted via the channel to the receiver. Then, the receiver produces the state estimate $\hat{X}_{t}$. The encoder uses (via feedback) the past channel outputs $\tilde{Z}_{0}^{t-1}$ or $\bar{Z}_{0}^{t-1}$ and/or the past channel inputs $Z_{0}^{t-1}$ to produce the current channel input $Z_{t}$. The decoder also uses all channel outputs $\tilde{Z}_{0}^{t}$ to produce $\hat{X}_{t}$. This system is subject to the conditional independence property. That is, $X_{t} \rightarrow$ $Z_{0}^{t} \rightarrow \tilde{Z}_{0}^{t} \rightarrow \hat{X}_{t}$ forms a Markov chain. From now on, we denote this communication system by the pair $\left(B_{\ell}, \Theta\right)$ to emphasize the uncertainty in the source and channel.

A necessary condition for uniform reliable data reconstruction capability of the communication system $\left(B_{\ell}, \Theta\right)$, in the sense that $E\left[\rho\left(X_{t}-\hat{X}_{t}\right)\right] \leq D, \forall \theta \in \Theta$, and $\forall \Gamma_{k} \in B_{\ell}(M(q \times q))(k \leq t-1)$, is given in the following theorem.

Theorem 3.4. Consider the communication system $\left(\left(B_{\ell}, \Theta\right)\right)$ as described above. For a given distortion level $D$, suppose that the limit, $\lim _{t \rightarrow \infty} \frac{1}{t+1} R_{t}(D)$, exists. Then, a necessary condition for the existence of an encoder and a decoder (estimator) for the uniform reliable data reconstruction is that the following inequality holds:

$$
\mathcal{C} \geq \lim _{t \rightarrow \infty} \frac{1}{t+1} R_{t}(D)
$$

Proof. See Appendix.

We have the following remarks regarding the above result.

\section{Remark 3.5.}

(i) The necessary condition (5) is independent of the class of encoders and therefore, it holds for all the encoders: Class A, Class B, and Class $C$;

(ii) The difference distortion measure $\rho(\cdot)$, used for the expression of the rate distortion $R_{t}(D)$, is 
chosen according to the desired reliable data reconstruction capability. For example, for moment reconstructability (i.e., $E\left\|X_{t}-\hat{X}_{t}\right\|^{r} \leq D$ ), we choose $\rho(x-\hat{x})=\rho_{r}(x-\hat{x})$.

By combining Lemma 3.3 and Theorem 3.4, we have the following necessary condition for UASAE.

Theorem 3.6. Consider the communication system $\left(B_{\ell}, \Theta\right)$, as described above. Suppose that $\lim _{t \rightarrow \infty}$ $\frac{1}{t+1} \sup _{P_{t}^{x} \in \mathcal{M}_{S, t}} H\left(p_{t}\right)$ exists. Then, a necessary condition for which UASAE holds is that the Shannon capacity $\mathcal{C}$ must satisfy the following inequality:

$$
\mathcal{C} \geq \lim _{t \rightarrow \infty} \frac{1}{t+1} \sup _{P_{t}^{x} \in \mathcal{M}_{S, t}} H\left(p_{t}\right) .
$$

Proof. See Appendix.

Corollary 3.7. Consider the communication system $\left(B_{\ell}, \Theta\right)$. Suppose that the initial state $X_{0}$ of the information source (1) has finite entropy. Then, a necessary condition for which UASAE holds is:

$$
\mathcal{C} \geq \sum_{i=1}^{q} \max \left\{0, \log \left|\lambda_{i}\left(A+\Gamma^{o}\right)\right|\right\},
$$

where:

$$
\begin{aligned}
\Gamma^{o} & \equiv \arg \max \{|\operatorname{det}(A+\Gamma)| \\
& \left.=\prod_{i=1}^{q}\left|\lambda_{i}(A+\Gamma)\right|, \Gamma \in B_{\ell}(M(q \times q))\right\} .
\end{aligned}
$$

Proof. See Appendix.

We have the following observation regarding the result of Corollary 3.7 .

\section{Remark 3.8.}

(i) Since the set $B_{\ell}(M(q \times q))$ is compact and $\operatorname{det}(\cdot)$ is a continuous function, there always exists $\Gamma^{\circ} \in$ $B_{\ell}(M(q \times q))$ satisfying Relation (7);

(ii) It is clear that the larger the $\ell$ is, the larger is the required capacity for UASAE to hold. In other words, the larger the system uncertainty is, the larger is the required capacity.

For the purpose of illustration of the necessary condition (7), we present the following example.

Example 3.9. Consider the CS shown in Figure 1. Suppose that the dynamic system is the uncertain system (1) with:

$$
A=\left(\begin{array}{cc}
-3 & 1 \\
0 & 2
\end{array}\right)
$$

and:

$$
\Gamma_{t}=\left(\begin{array}{cc}
\delta_{t} & 0 \\
0 & \gamma_{t}
\end{array}\right)
$$

where $\left\|\Gamma_{t}\right\| \leq \ell=1$ (recall that, for each $t \geq 0$, $\left\|\Gamma_{t}\right\| \equiv \sqrt{\lambda_{\max }\left(\Gamma_{t}^{\prime} \Gamma_{t}\right)}$, that is, $\max \left\{\left|\delta_{t}\right|,\left|\gamma_{t}\right|\right\} \leq 1$, $\forall t \in \mathbf{N}_{+}$). The channel is the compound erasure channel which, at each time step, transmits $\mathcal{R}$ bits in each channel use. The erasure probability $\theta$ is unknown and belongs to the set $\Theta=[0.1,0.5]$. Therefore, the capacity of this channel is $\mathcal{C}=0.5 \mathcal{R}$ (bits/time step). From Corollary 3.7, we have:

$$
\Gamma^{0}=\left(\begin{array}{cc}
-1 & 0 \\
0 & 1
\end{array}\right)
$$

and therefore, the necessary condition (7) is given by $0.5 \mathcal{R} \geq \log 12$. This means that for the rates $\mathcal{R}=$ $1,2,3,4,5,6,7$, we cannot find any encoder and decoder for which UASAE holds.

\section{Sufficient condition}

In this section, it is shown that the lower bound given by Relation (7) can also be a sufficient condition for UASAE of the nominal version of system (1) (i.e., $\ell=0$ ) over the compound erasure channels. This is shown by developing a differential coding strategy, which uses feedback channel with duty cycle $\beta \in$ $(0,1]$. Recall that $\beta=0$ implies non-availability of feedback channel and $\beta=1$ implies its full-time availability.

In this section, it is assumed that $\beta=\frac{M}{N}(M, N \in$ $\{1,2,3, \cdots\}, M \leq N)$, where for each $N$ updates on the state estimate, in the first $M$ updates, feedback channel is used in the encoder of Class C. Again, for simplicity of presentation, it is assumed that the measurement matrix $C$ in (1) is an identity matrix (if $C$ is not an identity matrix but $C^{\prime} C$ is invertible, then $\bar{Y}_{t} \equiv\left(C^{\prime} C\right)^{-1} C^{\prime} Y_{t}=X_{t}$ is treated as the observation signal). Moreover, it is assumed that the encoder and decoder are aware of policies of each other and $X_{0}$ has a bounded support in $\mathbf{R}^{q}$.

The differential coding strategy is described in the proof of the following proposition, which shows that UASAE holds for System (1) over the compound erasure channel.

Proposition 4.1. Consider the communication system $\mathbf{R}^{q}$ whose capacity is given by $\mathcal{C}=\inf _{\theta \in \Theta}(1-\theta) \mathcal{R}$, where $\mathcal{R}$ is the average transmission bit rate. Suppose that the information source is given by the nominal version of System (1) (i.e., $\ell=0$ ) and the encoder is of Class $C$. Let $X_{0}$ have a bounded support and $C^{\prime} C$ be invertible. Then, a sufficient condition for which UASAE holds is that the capacity $\mathcal{C}$ (measured in bits/time step) satisfies the following lower bound: 


$$
\mathcal{C}>\sum_{i=1}^{q} \max \left\{0, \log \left|\lambda_{i}(A)\right|\right\}=\lim _{t \rightarrow \infty} \frac{1}{t+1} H\left(p_{t}\right) .
$$

Proof. See Appendix.

We have the following observation regarding the above result.

\section{Remark 4.2.}

(i) The sufficient condition (8) implies that there exists a coding strategy for which UASAE holds for the nominal version of System (1) over the compound erasure channel that uses feedback channel with duty cycle, $\beta \in(0,1]$. This strategy results in UASAE if the transmission rate $\mathcal{R}$ is greater than the rate:

$$
\frac{1}{\inf _{\theta \in \Theta}(1-\theta)} \sum_{i=1}^{q} \max \left\{0, \log \left|\lambda_{i}(A)\right|\right\},
$$

(ii) From Corollary 3.7, it follows that the eigenvalues rate condition described by the Shannon capacity is tight (i.e., the necessary and sufficient condition) for which UASAE holds for the nominal version of System (1) over the compound erasure channel. In other words, the eigenvalues rate:

$$
\sum_{i=1}^{q} \max \left\{0, \log \left|\lambda_{i}(A)\right|\right\}
$$

is the minimum capacity required for UASAE;

(iii) In this paper, we have been concerned with uncertainty in communication channel; and as we have shown above, in the presence of uncertainty in the channel, the eigenvalues rate condition described by the Shannon capacity is tight for almost sure estimation.

When a communication channel has limited capacity, it is desirable to have estimation using the minimum possible capacity. As shown above, the minimum required capacity for almost sure estimation equals the eigenvalues rate:

$$
\sum_{i=1}^{q} \max \left\{0, \log \left|\lambda_{i}(A)\right|\right\}
$$

In the problem considered in this paper, the information source (dynamic system) is given; therefore, the eigenvalues rate is fixed and, in many cases, noninteger. However, non-integer rate:

$$
\frac{M}{N} \frac{1}{\inf _{\theta \in \Theta}(1-\theta)} \sum_{i=1}^{q} \max \left\{0, \log \left|\lambda_{i}(A)\right|\right\},
$$

cannot be put on communication channel. Hence, all we can do is proper modification of the proposed coding strategy to achieve almost sure estimation by the use of the minimum required capacity. This modification is described below.

Consider a small positive real number, $\eta$, and define $\mathcal{R}_{\min }$ by the following expression:

$$
\begin{aligned}
\mathcal{R}_{\min } \equiv & \frac{M}{N} \frac{1}{\inf _{\theta \in \Theta}(1-\theta)} \sum_{i=1}^{q} \max \left\{0, \log \left|\lambda_{i}(A)\right|\right\} \\
& +\frac{\eta}{\inf _{\theta \in \Theta}(1-\theta)} .
\end{aligned}
$$

If $\mathcal{R}_{\text {min }}$ is an integer number, this rate can be put into the channel by implementing the proposed differential coding strategy; therefore, we have UASAE by transmission with the minimum required capacity. However, in general, $\mathcal{R}_{\min }$ may not be an integer number. For this case, we use the following time-sharing strategy to achieve UASAE by the use of the minimum required capacity.

\subsection{Time-sharing strategy}

For simplicity, first, consider the scalar case and without loss of generality, suppose $M=N=1$. Let $i \in \mathbf{N}_{+}$ be the smallest integer such that $i \leq \mathcal{R}_{\min }<1+i$. Also, let $\xi \equiv \mathcal{R}_{\min }-i$. Suppose that both rates $i$ and $1+i$ can be put into the channel. Then, unlike the proposed differential coding strategy, the encoder now partitions the box $\left[-L_{t}, L_{t}\right]$, wherein the estimation error lies, into $2^{\mathcal{R}_{t}}$ equal-size non-overlapping intervals, where $\mathcal{R}_{t}$ takes values from the set $\mathcal{R}_{t} \in\{i, 1+i\}$ according to the following time-sharing strategy:

$$
\left\{\begin{array}{l}
P\left(\mathcal{R}_{t}=i\right)=1-\xi \\
P\left(\mathcal{R}_{t}=1+i\right)=\xi
\end{array}\right.
$$

Subsequently, by the strong law of large numbers, the average transmitted rate denoted here by $\mathcal{R}^{a v} \equiv \lim _{t \rightarrow \infty} \frac{1}{t+1} \sum_{j=0}^{t} \mathcal{R}_{j}$ equals $E\left[\mathcal{R}_{0}\right]$ almost surely, where:

$$
\begin{aligned}
E\left[\mathcal{R}_{0}\right] & =(1-\xi) i+\xi(1+i)=i-\xi i+\xi+\xi i \\
& =i+\mathcal{R}_{\min }-i=\mathcal{R}_{\min } .
\end{aligned}
$$

In other words, here, we are transmitting information with the minimum required capacity:

$$
\begin{aligned}
\mathcal{C} & =\inf _{\theta \in \Theta}(1-\theta) \mathcal{R}^{a v}=\inf _{\theta \in \Theta}(1-\theta) \mathcal{R}_{\text {min }} \\
& =\sum_{i=1}^{q} \max \left\{0, \log \left|\lambda_{i}(A)\right|\right\}+\eta .
\end{aligned}
$$

Now, we must also show that for this time-sharing strategy, UASAE holds. By implementing the proposed coding strategy and noting that here, the transmission rate is specified by the above time-sharing law, 
we have $\left|X_{t}-\hat{X}_{t}\right| \leq V_{t}$, where the random variables, $F_{t}$, are i.i.d. with common distribution given by:

$$
\begin{aligned}
& P\left(F_{t}=1\right)=\theta, \\
& P\left(F_{t}=2^{-i}\right)=(1-\xi)(1-\theta), \\
& P\left(F_{t}=2^{-(1+i)}\right)=\xi(1-\theta) .
\end{aligned}
$$

Subsequently, along the same lines of the proof of Proposition 4.1, it is shown that using the above timesharing strategy $V_{t} \rightarrow 0$; therefore, UASAE holds. Note that when the erasure channel transmits a packet of data, $\mathcal{R}_{t}$, successfully, from the number of received bits, the decoder can identify whether $\mathcal{R}_{t}=i$ or $\mathcal{R}_{t}=1+i$ has been selected by the encoder as the communication channel is assumed to be ternary with input and output 0,1 , idle. This type of channel can be obtained, for example, by implementing an Amplitude Shift Keying (ASK) type modulation scheme, in which when the amplitude of the sampled received signal is above $V(V>0)$, it is decoded as 1 and when it is less than $-V$, it is decoded as 0 ; otherwise, the channel is assumed to be in the idle mode. Consequently, at each time instant, by counting the number of the received 0 and 1 bits, the length of the received packet can be identified. Hence, the decoder can identify which one $(i$ or $1+i$ ) has been chosen for transmission. Another way for the decoder to know the length of transmitted bits is to choose $i$ or $1+i$ deterministically, instead of randomly.

Extension of the above strategy to the vector case is straightforward. It is realized by implementing a similarity transformation that turns the system matrix A into the real Jordan form, noting that now the transmission rate is defined following a time-sharing strategy, as specified below:

Let $\lambda_{k}(A)$ be the eigenvalue of the system matrix $A$ corresponding to the $k$ th $(k=1,2, \cdots, m, m \leq q)$ Jordan block of the matrix $A \in M(q \times q)$. For each $\lambda_{k}(A)$, let $i_{k}$ be the smallest integer such that:

$$
\begin{aligned}
i_{k} \leq \mathcal{R}_{\min }^{(k)} & \equiv \max \left\{0, \frac{1}{\inf _{\theta \in \Theta}(1-\theta)} \log \left|\lambda_{k}(A)\right|\right\} \\
& +\frac{\eta}{\inf _{\theta \in \Theta}(1-\theta)}<1+i_{k} .
\end{aligned}
$$

Also, let $\xi_{k}=\mathcal{R}_{\min }^{(k)}-i_{k}$. Accordingly, for each $\lambda_{k}(A)$, the encoder partitions the box associated with $\lambda_{k}(A)$ into $2^{\mathcal{R}_{t}^{(k)}}$ equal-size non-overlapping intervals, where $\mathcal{R}_{t}^{(k)}$ is chosen according to the following i.i.d. distribution:

$$
\left\{\begin{array}{l}
P\left(\mathcal{R}_{t}^{(k)}=i_{k}\right)=1-\xi_{k}, \\
P\left(\mathcal{R}_{t}^{(k)}=1+i_{k}\right)=\xi_{k} .
\end{array}\right.
$$

\subsection{Simulation result}

The results shown in Figure 2 illustrate the performance of the proposed time-sharing strategy when the communication is via the compound erasure channel, which uses feedback channel all the time (i.e., $\beta=1)$. Here, it is assumed that the unknown erasure probability, $\theta$, belongs to the set $\Theta=[0.1,0.5]$. The dynamic system (1) is assumed to be scalar with $A=$ -3 and $\ell=0$. The initial condition $X_{0}$ is uniformly distributed in the interval $[-1,1]$, i.e., $L_{0}=1$. From Corollary 3.7 and Proposition 4.1, it follows that $\frac{1}{\inf _{\theta \in \Theta}(1-\theta)} \log |A|=\frac{1}{0.5} \log 3=3.1699$ (bits/time step) is the minimum transmission rate for which UASAE holds. However, this rate is not an integer number. Therefore, to have almost sure estimation requiring the minimum capacity $\mathcal{C}=\log |A|=1.58$ (bits/time step) for the case of $\beta=1$, we need to implement the proposed time-sharing strategy. Figure 2 illustrates the performance of the proposed strategy for $A=-3$, $\ell=0, L_{0}=1, \Theta=[0.1,0.5]$ and $\eta=0.0005$. It is clear from Figure 2 that although the erasure probability, $\theta$, is unknown, after a few iterations, the absolute value of the estimation error (i.e., $\left.\left|X_{t}-\hat{X}_{t}\right|\right)$ converges to zero, while the average transmission rate, $\mathcal{R}^{a v}=$ $\lim _{t \rightarrow \infty} \frac{1}{t+1} \sum_{j=0}^{t} \mathcal{R}_{j}$, equals the minimum required transmission rate $\frac{1}{\inf _{\theta \in \Theta}(1-\theta)} \log |A|$. That is, here, the capacity $\mathcal{C} \equiv \inf _{\theta \in[0.1,0.5]}(1-\theta) \mathcal{R}^{a v}$ is going to equal the minimum required capacity $1: 58$ (bits/time step).

In addition, it is observed that if the above technique is applied to the case where $\theta \in \bar{\Theta} \subset \Theta$, the estimation error is asymptotically zero. If $\bar{\Theta}_{1}, \bar{\Theta}_{2} \subset \Theta$ where each point in $\bar{\Theta}_{2}$ is greater than all the points in $\bar{\Theta}_{1}$, the performance of the case of $\theta \in \bar{\Theta}_{1} \subset \Theta$ is better than the performance of the case of $\theta \in \bar{\Theta}_{2} \subset \Theta$, as expected. Also, if $\theta \in \widetilde{\Theta}$, where each point in $\widetilde{\Theta}$ is greater than all points in $\Theta$, the performance is poor and in some cases, the estimation error may become unbounded.

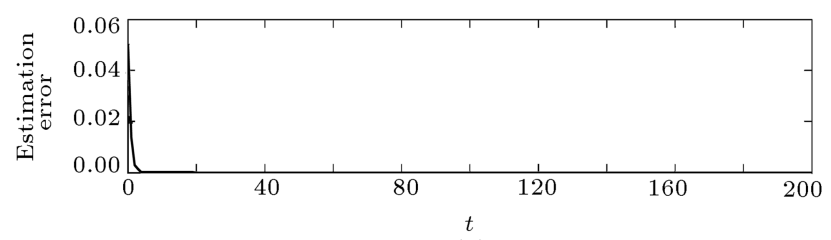

(a)

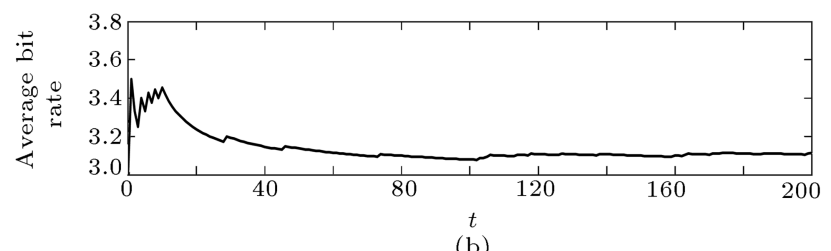

(b)

Figure 2. Simulation result for $\beta=1, A=-3, \ell=0$, $L_{0}=1, \Theta=[0.1,0.5]$, and $\eta=0.0005$ : (a) Estimation error $\left|X_{t}-\hat{X}_{t}\right|$, and (b) the average bit rate. 


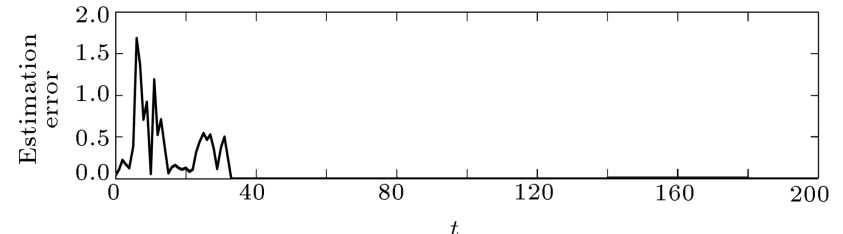

(a)

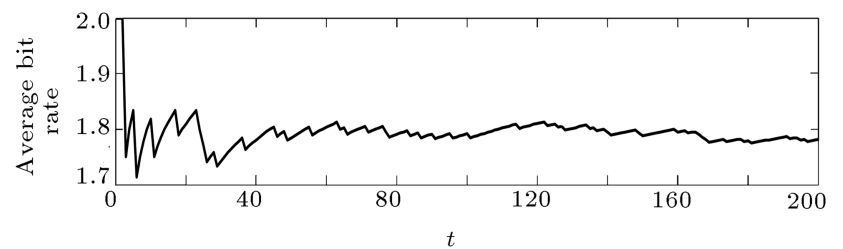

(b)

Figure 3. Simulation result for $\beta=1, A=-3, \ell=0$, $L_{0}=1, \theta=0.1$, and $\eta=0.0005$ : (a) Estimation error $\left|X_{t}-\hat{X}_{t}\right|$, and (b) the average bit rate.

Figure 3 illustrates the performance of the proposed coding and time-sharing strategy when the erasure probability $\theta$ is known and equivalent to $\theta=0.1$ and $\beta=1$. As it is clear in Figure 3, the proposed strategy is able to estimate the state of the system with asymptotically zero estimation error by transmission with the minimum rate of 1.7611 bits/time step. However, the performance for this case is not as good as the performance of the other case (Figure 2). This is due to the fact that, as shown in Figure 2, by taking a conservative approach, more bits are transmitted, which results in a better performance.

\section{Conclusion}

This paper extended the previous results to cover the cases in which both the dynamic system and communication channel were uncertain, and the feedback channel had a duty cycle, $\beta \in(0,1]$. A necessary condition in which UASAE held was derived under this general situation. Moreover, a sufficient condition was presented in which UASAE held over the compound erasure channels when the dynamic system was not uncertain, but the channel was. Consequently, it was concluded that over the compound erasure channel, a condition for the Shannon capacity in terms of the rate of expansion of the Shannon entropy would be necessary and sufficient for uniform almost sure asymptotic zero estimation error. Possible extension for future includes addressing the estimation problem of stochastic nonlinear uncertain dynamic systems over noisy communication channels, which use feedback links with a Markov chain model.

\section{References}

1. Brinon Arranz, L., Seuret, A., and Canudas de Wit, C. "Translation control of a fleet circular formation of AUVs under finite communication range", In Proc. 48th IEEE Conference on Decision and Control, pp. 8345-8350 (2009).

2. Brinon Arranz, L., Seuret, A., and Canudas de Wit, C. "Collaborative estimation of gradient direction by a formation of AUVs under communication constraints", In Proceedings of the 50th IEEE Conference on Decision and Control, pp. 5583-5588 (2011).

3. Elia, N. "When bode meets Shannon: control-oriented feedback communication schemes", IEEE Trans. Automat. Contr., 49(9), pp. 1477-1488 (2004).

4. Elia, N. and Eisenbeis, J.N. "Limitations of linear control over packet drop networks", IEEE Trans. Automat. Contr., 56(4), pp. 826-841 (2011).

5. Martins, N.C., Dahleh, A., and Elia, N. "Feedback stabilization of uncertain systems in the presence of a direct link", IEEE Trans. Automat. Contr., 51(3), pp. 438-447 (2006).

6. Minero, P., Franceschetti, M., Dey, S., and Nair, N. "Data rate theorem for stabilization over time-varying feedback channels", IEEE Trans. Automat. Contr., 54(2), pp. 243-255 (Feb., 2009).

7. Minero, P., Coviello, L., and Franceschetti, M. "Stabilization over Markov feedback channels: the general case", IEEE Trans. Automat. Contr., 58(2), pp. 349362 (2013).

8. Nair, G.N., Evans, R.J., Mareels, I.M.Y., and Moran, W. "Topological feedback entropy and nonlinear stabilization", IEEE Trans. Automat. Contr., 49(9), pp. 1585-1597 (2004).

9. Nair, G.N. and Evans, R.J. "Stabilizability of stochastic linear systems with finite feedback data rates", SIAM J. Control Optimization, 43(3), pp. 413-436 (2004).

10. Canudas de Wit, C., Gomez-Estern, F., and Rodrigues Rubio, F. "Delta-modulation coding redesign for feedback-controlled systems", IEEE Transactions on Industrial Electronics, 56(7), pp. 2684-2696 (2009).

11. Farhadi, A. and Ahmed, N.U. "Tracking nonlinear noisy dynamic systems over noisy communication channels", IEEE Transactions on Communications, 59(4), pp. 955-961 (2011).

12. Charalambous, C.D. and Farhadi, A. "A mathematical framework for robust control over uncertain communication channels", Proceedings of the 44th IEEE Conference on Decision and Control, Seville, Spain, pp. 2530-2535 (2005).

13. Charalambous, C.D. and Farhadi, A. "Control of feedback systems subject to the finite rate constraints via Shannon lower bound", Proceedings of the 5th International Symposium on Modeling and Optimization in Mobile, Ad Hoc, and Wireless Networks, Cyprus, pp. 1-7 (2007).

14. Farhadi, A., Domun, J., and Canudas de Wit, C. "A supervisory control policy over an acoustic communication network", International Journal of Control, 88(5), pp. 946-958 (2015). 
15. Farhadi, A. "Feedback channel in linear noiseless dynamic systems controlled over the packet erasure network", International Journal of Control, 88(8), pp. 1490-1503 (2015).

16. Farhadi, A. "Stability of linear dynamic systems over the packet erasure channel: a co-design approach", International Journal of Control, 88(12), pp. 24882498 (2015).

17. Farhadi, A. "Stability of nonlinear uncertain Lipschitz systems over the digital noiseless channel", Scientia Iranica, Transactions on Computer Science and Engineering and Electrical Engineering, 25(3), pp. 1523 1532 (2018).

18. Tatikonda, S. and Mitter, S. "Control over noisy channels", IEEE Transactions on Automatic Control, 49(7), pp. 1196-1201 (2004).

19. Jiang, X.W., Guan, Z.H., Feng, G., Wu, Y., and Yuan, F.S. "Optimal tracking performance of networked control systems with channel input power constraint", IET Control Theory and Applications, 6(11), pp. 16901698 (2012).

20. Zhan, X.S., Wu, J., Jiang, T., and Jiang, X.W. "Optimal performance of networked control systems under the packet dropouts and channel noise", ISA Transactions, 58, pp. 214-221 (2015).

21. Jiang, X.W., Hu, B., Guan, Z.H., Zhang, X.H., and $\mathrm{Yu}, \mathrm{L}$. "Best achievable tracking performance for networked control systems with encoder-decoder", Information Sciences, 305, pp. 184-195 (2015).

22. Matveev, A.S. and Savkin, A.V. "Shannon zero error capacity in the problems of state estimation and stabilization via noisy communication channels", International Journal of Control, 80, pp. 241-255 (2007).

23. Cover, T.M. and Thomas, J.A., Elements of Information Theory, John Wiley and Sons, USA (1991).

24. Geromel, J.C. "Optimal linear filtering under parameter uncertainty", IEEE Transactions on Signal Processing, 47(1), pp. 168-175 (1999).

25. Xu, S., Dooren, P.V., Stefan, R., and Lam, J. "Robust stability and stabilization for singular systems with state delay and parameter uncertainty", IEEE Transactions on Automatic Control, 47(7), pp. 1122-1128 (2002).

26. Gao, H., Meng, X., and Chen, T. "A parameterdependent approach to robust $H_{\infty}$ filtering for timedelay systems", IEEE Transactions on Automatic Control, 53(10), pp. 2420-2425 (2008).

27. El Ghaoui, L. and Calafiore, G. "Robust filtering for discrete-time systems with bounded noise and parametric uncertainty", IEEE Transactions on Automatic Control, 46(7), pp. 1084-1089 (2001).

28. Wolfowitz, J. "Simultaneous channels", Archive for Rational Mechanics and Analysis, 4, pp. 371-386 (1959).

29. Lapidoth, A. and Narayan, P. "Reliable communication under channel uncertainty", IEEE Transactions on Information Theory, 44(6), pp. 2148-2177 (1998).
30. Shrader, B. and Permuter, H. "Feedback capacity of the compound channel", IEEE Transactions on Information Theory, 55(8), pp. 3629-3644 (2009).

31. Sakrison, D.J. "The rate distortion function for a class of sources", Information and Control, 15, pp. 165-195 (1969).

32. Linder, T. and Zamir, R. "On the asymptotic tightness of the Shannon lower bound", IEEE Transactions on Information Theory, 40(6), pp. 2026-2031 (1994).

33. Charalambous, C.D. and Farhadi, A. "LQG optimality and separation principle for general discrete time partially observed stochastic systems over finite capacity communication channels", Automatica, 44(12), pp. 3181-3188 (2008).

34. Billingsley, P. Probability and Measure, John Wiley and Sons, USA (1995).

\section{Appendix}

\section{Proof of Lemma 3.3.}

(i) The first part is well known and for the detailed proof, see [32];

(ii) From the classical Shannon lower bound, as given in part (i), it follows that for each $P_{t}^{x} \in$ $\mathcal{M}_{S, t}$, we have $\inf _{Q_{t} \in \mathcal{M}_{D}\left(P_{t}^{x}\right)} I\left(X_{t}, \hat{X}_{t}\right) \geq H\left(p_{t}\right)-$ $\max _{h \in G_{D}} H(h)$. Consequently, the robust rate distortion $R_{t}(D)$ satisfies the following inequalities:

$$
\begin{aligned}
R_{t}(D) & \equiv \inf _{Q_{t} \in \mathcal{M}_{D}\left(P_{t}^{x}\right)} \sup _{P_{t}^{x} \in \mathcal{M}_{S, t}} I\left(X_{t}, \hat{X}_{t}\right) \\
& \geq \sup _{P_{t}^{x} \in \mathcal{M}_{S, t}} \inf _{Q_{t} \in \mathcal{M}_{D}\left(P_{t}^{x}\right)} I\left(X_{t}, \hat{X}_{t}\right) \\
& \geq \sup _{P_{t}^{x} \in \mathcal{M}_{S, t}}\left(H\left(p_{t}\right)-\max _{h \in G_{D}} H(h)\right) .
\end{aligned}
$$

Now, as $G_{D}$ is independent of $P_{t}^{x}$, from the above expression, we have:

$$
R_{t}(D) \geq \sup _{P_{t}^{x} \in \mathcal{M}_{S, t}} H\left(p_{t}\right)-\max _{h \in G_{D}} H(h) .
$$

This completes the proof.

Proof of Theorem 3.4. Let $\left\{\bar{P}_{0, t}^{z}\right\}$ denote the set of all distributions corresponding to the channel input sequence $Z_{0}^{t}$ when the source message $X_{t}$ with distribution $P_{t}^{x} \in \mathcal{M}_{S, t}$ is transmitted. Suppose that there exists an encoder-decoder pair such that we have the uniform reliable data reconstruction. Then, it follows from the data processing inequality [23], as described by $I^{\theta}\left(Z_{0}^{t}, \tilde{Z}_{0}^{t}\right) \geq I^{\theta}\left(X_{t}, \hat{X}_{t}\right)\left(\forall P_{t}^{x} \in \mathcal{M}_{S, t}\right.$ and $\forall \theta \in \Theta)$, that, for a given $\theta \in \Theta$, we have:

$$
\sup _{\left\{\bar{P}_{0, t}^{z}\right\}} I^{\theta}\left(Z_{0}^{t}, \tilde{Z}_{0}^{t}\right) \geq \sup _{P_{t}^{x} \in \mathcal{M}_{S, t}} I^{\theta}\left(X_{t}, \hat{X}_{t}\right) \text {. }
$$


Let $\left\{P_{0, t}^{z}\right\}$ denote the set of all channel input distributions. Evidently, $\left\{\bar{P}_{0, t}^{z}\right\} \subseteq\left\{P_{0, t}^{z}\right\}$ and hence:

$$
\sup _{P_{0, t}^{z} \in \mathcal{M}_{1}\left(\mathcal{Z}_{0, t}\right)} I^{\theta}\left(Z_{0}^{t}, \tilde{Z}_{0}^{t}\right) \geq \sup _{\left\{\bar{P}_{0, t}^{z}\right\}} I^{\theta}\left(Z_{0}^{t}, \tilde{Z}_{0}^{t}\right)
$$

Therefore, for a given $\theta \in \Theta$, it follows from Relation (A.1) that:

$$
\begin{aligned}
\sup _{P_{0, t}^{z} \in \mathcal{M}_{1}\left(\mathcal{Z}_{0, t}\right)} I^{\theta}\left(Z_{0}^{t}, \tilde{Z}_{0}^{t}\right) & \\
\geq & \inf _{Q_{t}^{\theta} \in \mathcal{M}_{D}\left(P_{t}^{x}\right)} \sup _{P_{t}^{x} \in \mathcal{M}_{S, t}} I^{\theta}\left(X_{t}, \hat{X}_{t}\right),
\end{aligned}
$$

where $Q_{t}^{\theta}$ is the stochastic kernel corresponding to $X_{t}$ and $\hat{X}_{t}$ given $\theta \in \Theta$. By definition:

$$
\begin{aligned}
& I^{\theta}\left(X_{t}, \hat{X}_{t}\right) \\
& \quad \equiv \iint \log \left(\frac{Q_{t}^{\theta}(d \hat{x} \mid x)}{\int Q_{t}^{\theta}(d \hat{x} \mid x) P_{t}^{x}(d x)}\right) Q_{t}^{\theta}(d \hat{x} \mid x) P_{t}^{x}(d x)
\end{aligned}
$$

Evidently, infimum of the term $\sup _{P_{t}^{x} \in \mathcal{M}_{S, t}} I^{\theta}\left(X_{t}, \hat{X}_{t}\right)$ with respect to $Q_{t}^{\theta} \in \mathcal{M}_{D}\left(P_{t}^{x}\right)$ is independent of $Q_{t}^{\theta}$. Hence:

$$
\inf _{Q_{t}^{\theta} \in \mathcal{M}_{D}\left(P_{t}^{x}\right)} \sup _{P_{t}^{x} \in \mathcal{M}_{S, t}} I^{\theta}\left(X_{t}, \hat{X}_{t}\right)
$$

is independent of $\theta$. That is:

$$
\inf _{Q_{t}^{\theta} \in \mathcal{M}_{D}\left(P_{t}^{x}\right)} \sup _{P_{t}^{x} \in \mathcal{M}_{S, t}} I^{\theta}\left(X_{t}, \hat{X}_{t}\right)=R_{t}(D) .
$$

Therefore, by taking infimum with respect to $\theta$ over the set $\Theta$, it follows from Relations (A.2) and (A.3) that the following inequality holds:

$$
\inf _{\theta \in \Theta} \sup _{P_{0, t}^{z} \in \mathcal{M}_{1}\left(\mathcal{Z}_{0, t}\right)} I^{\theta}\left(Z_{0}^{t}, \tilde{Z}_{0}^{t}\right) \geq R_{t}(D) .
$$

Hence, it follows from Definition 3.1 and Eq. (2) that:

$$
\begin{aligned}
\mathcal{C}_{t} & \equiv \sup _{P_{0, t}^{z} \in \mathcal{M}_{1}\left(\mathcal{Z}_{0, t}\right)} \inf _{\theta \in \Theta} I^{\theta}\left(Z_{0}^{t}, \tilde{Z}_{0}^{t}\right) \\
& =\inf _{\theta \in \Theta} \sup _{P_{0, t}^{z} \in \mathcal{M}_{1}\left(\mathcal{Z}_{0, t}\right)} I^{\theta}\left(Z_{0}^{t}, \tilde{Z}_{0}^{t}\right) \geq R_{t}(D) .
\end{aligned}
$$

Therefore, under the assumption of the existence of an encoder-decoder pair that yields an average distortion $E\left[\rho\left(X_{t}-\hat{X}_{t}\right)\right] \leq D$, for all $\Gamma_{k} \in B_{\ell}(M(q \times q))(k \leq t-1)$ and $\forall \theta \in \Theta$, we have $\mathcal{C}_{t} \geq R_{t}(D)$, and therefore:

$$
\mathcal{C} \equiv \lim _{t \rightarrow \infty} \frac{1}{t+1} \mathcal{C}_{t} \geq \lim _{t \rightarrow \infty} \frac{1}{t+1} R_{t}(D)
$$

That is, $\mathcal{C} \geq \lim _{t \rightarrow \infty} \frac{1}{t+1} R_{t}(D)$ is a necessary condition for the existence of an encoder-decoder pair. This completes the proof.
Proof of Theorem 3.6. Suppose that UASAE holds. This implies that for any $\epsilon>0$, there exists a $T(\epsilon)<\infty$ such that the following inequality holds:

$$
\begin{aligned}
& P\left(\sup _{t \geq T(\epsilon)}\left\|X_{t}-\hat{X}_{t}\right\|>\epsilon\right) \leq \epsilon, \\
& \forall \Gamma_{k} \in B_{\ell}(M(q \times q))(k \leq t-1), \\
& \forall \theta \in \Theta .
\end{aligned}
$$

Now, if we choose $\rho(\cdot)$ as the indicator measure, i.e.:

$$
\rho(\xi)=\rho_{\epsilon}(\xi)=\left\{\begin{array}{ll}
0 & \text { if }|| \xi|| \leq \epsilon \\
1 & \text { if }|| \xi \|>\epsilon
\end{array} \quad \text { for } t \geq T(\epsilon),\right.
$$

we have:

$$
E\left[\rho\left(X_{t}-\hat{X}_{t}\right)\right]=P\left(\left\|X_{t}-\hat{X}_{t}\right\|>\epsilon\right) \leq \epsilon,
$$

uniformly with respect to $B_{\ell}(M(q \times q))$ and $\Theta$. Therefore, from Theorem 3.4 and Lemma 3.3, the capacity and robust rate distortion must, for all $t \geq T(\epsilon)$, satisfy the following inequalities:

$$
\begin{aligned}
\frac{1}{t+1} \mathcal{C}_{t} \geq & \frac{1}{t+1} R_{t}(\epsilon) \geq \frac{1}{t+1} \sup _{P_{t}^{x} \in \mathcal{M}_{S, t}} H\left(p_{t}\right) \\
& -\frac{1}{t+1} \max _{h \in G_{\epsilon}} H(h) .
\end{aligned}
$$

It is known that for the indicator distortion measure, $\max _{h \in G_{\epsilon}} H(h)$ is finite [33]. Hence, it follows from the above expression that:

$$
\mathcal{C} \equiv \lim _{t \rightarrow \infty} \frac{1}{t+1} \mathcal{C}_{t} \geq \lim _{t \rightarrow \infty} \frac{1}{t+1} \sup _{P_{t}^{x} \in \mathcal{M}_{S, t}} H\left(p_{t}\right) .
$$

This proves that Inequality (6) is a necessary condition for UASAE.

Proof of Corollary 3.7. From Theorem 3.6, we have the following inequality as a necessary condition for UASAE:

$$
\mathcal{C} \geq \lim _{t \rightarrow \infty} \frac{1}{t+1} \sup _{P_{t}^{x} \in \mathcal{M}_{S, t}} H\left(p_{t}\right),
$$

where the density function $p_{t}$ is induced by R.V. $X_{t}=$ $\left(\prod_{t-1 \geq k \geq 0}\left(A+\Gamma_{k}\right)\right) X_{0}$. Hence, it follows from ([23], p. 234) that:

$$
H\left(p_{t}\right)=\log \left|\operatorname{det}\left(\prod_{t-1 \geq k \geq 0}\left(A+\Gamma_{k}\right)\right)\right|+H\left(p_{0}\right) .
$$

Therefore:

$$
\sup _{P_{t}^{x} \in \mathcal{M}_{S, t}} H\left(p_{t}\right)=H\left(p_{0}\right)
$$




$$
\sup _{\left\{|| \Gamma_{k} \| \leq l, 0 \leq k \leq t-1\right\}}\left(\log \left|\operatorname{det}\left(\prod_{t-1 \geq k \geq 0}\left(A+\Gamma_{k}\right)\right)\right|\right) .
$$

Consequently:

$$
\begin{aligned}
\lim _{t \rightarrow \infty} \frac{1}{t+1} \sup _{P_{t}^{x} \in \mathcal{M}_{S, t}} H\left(p_{t}\right) & =\lim _{t \rightarrow \infty} \frac{1}{t+1} \\
& \sup _{\left\{|| \Gamma_{k} \| \leq l, 0 \leq k \leq t-1\right\}}\left(\log \left|\operatorname{det}\left(\prod_{t-1 \geq k \geq 0}\left(A+\Gamma_{k}\right)\right)\right|\right)
\end{aligned}
$$

We can always find a similarity transformation $T$ such that the matrix $A+\Gamma^{o}$ can be written in the following form:

$$
T^{-1}\left(A+\Gamma^{o}\right) T=\left(\begin{array}{cc}
\left(A+\Gamma^{o}\right)_{s} & \\
& \left(A+\Gamma^{o}\right)_{u s}
\end{array}\right),
$$

where $\left(A+\Gamma^{o}\right)_{s}$ has eigenvalues inside the unit circle and $\left(A+\Gamma^{o}\right)_{u s}$ has eigenvalues on or outside the unit circle. Accordingly, we split the state space into the following two disjoint subspaces:

(i) Stable subspace, which corresponds to $\left(A+\Gamma^{o}\right)_{s}$;

(ii) Unstable subspace, which corresponds to $(A+$ $\left.\Gamma^{o}\right)_{u s}$.

Let $\mathcal{P}_{S}$ be the projection onto the stable subspace. Then, $\lim _{t \rightarrow \infty} \mathcal{P}_{S}\left(X_{t}\right)=0$. Hence, for sufficiently large $t$, the projection of the state onto the stable subspace is reconstructed as zero. That is, for large $t$, the stable subspace does not contribute to the entropy of the R.V. $X_{t}$. Therefore, without loss of generality, in computing the entropy of the R.V. $X_{t}$, we can restrict our attention to the matrix $A+\Gamma^{o}$ that contains only unstable eigenvalues.

In view of this fact, it follows from Eq. (12) that:

$$
\begin{aligned}
\lim _{t \rightarrow \infty} & \frac{1}{t+1} \sup _{P_{t}^{x} \in \mathcal{M}_{S, t}} H\left(p_{t}\right)=\log \left|\operatorname{det}\left(A+\Gamma^{o}\right)\right|_{u s} \\
\quad & \log \prod_{i=1}^{q}\left|\lambda_{i}\left(A+\Gamma^{o}\right)_{u s}\right|=\sum_{i=1}^{q} \log \left|\lambda_{i}\left(A+\Gamma^{o}\right)_{u s}\right| .
\end{aligned}
$$

Therefore, for the matrix $A+\Gamma^{o}$ (with some stable eigenvalues), we have the following necessary condition for UASAE:

$$
\mathcal{C} \geq \sum_{i=1}^{q} \max \left\{0, \log \left|\lambda_{i}\left(A+\Gamma^{o}\right)\right|\right\} .
$$

This completes the proof.

Proof of Proposition 4.1. In what follows, we consider the scalar system. Extension of the results to the general vector case is straightforward and it follows by implementing a similarity transformation that turns the system matrix $A$ to the real Jordan form [18].

As the initial state is bounded, we have $\left|X_{0}\right| \leq$ $L_{0}$, where $L_{0}$ is known a priori. At time instant $t=0$, the encoder partitions the interval $\left[-L_{0}, L_{0}\right]$ into $2^{\frac{N}{M} \mathcal{R}}$ equal-size bins, and upon observing $X_{0}$, it identifies the bin where $X_{0}$ is located, and represents the corresponding index by $\frac{N}{M} \mathcal{R}$ bits and transmits the corresponding packet. Then, the output of the decoder is updated by Eq. (A.6):

$$
\hat{X}_{t}= \begin{cases}\gamma_{j}+\hat{X}_{t}^{e} & \text { if erasure does not occur } \\ \hat{X}_{t}^{e} & \text { if erasure occurs }\end{cases}
$$

where $\gamma_{j}$ is the center of the $j+1$ bin, which contains $X_{t}-\hat{X}_{t}^{e}$. Note that $\hat{X}_{0}^{e}=0$ and the encoder and decoder are aware of each other's policies when the feedback channel is available; hence, the decoder can determine $\hat{X}_{t}^{e}$ when feedback channel is available. Consequently, for the time instant $t=0$, the decoding error is bounded above by:

$$
\left|X_{0}-\hat{X}_{0}\right| \leq V_{0}
$$

where:

$$
V_{0}= \begin{cases}\frac{L_{0}}{2^{\frac{N}{M} \mathcal{R}}} & \text { if erasure does not occur } \\ L_{0} & \text { if erasure occurs }\end{cases}
$$

At time instant $t=1$, using feedback channel, the encoder can determine $V_{0}$ and $\hat{X}_{0}$. Subsequently, it computes $\hat{X}_{1}^{e}=A \hat{X}_{0}$ and $L_{1}=|A| V_{0}$ (note that during the time period between two time instants $t=0$ and $t=1$, feedback channel is used). Then, it partitions the interval $\left[-L_{1}, L_{1}\right]$ into $2^{\frac{N}{M} \mathcal{R}}$ bins. Upon observing $X_{1}$, the encoder computes $X_{1}-\hat{X}_{1}^{e}$ and determines the bin, where $X_{1}-\hat{X}_{1}^{e}$ is located. Then, it represents the index of this bin by $\frac{N}{M} \mathcal{R}$ bits and transmits the corresponding packet. Subsequently, the decoder output is updated by Eq. (A.6). For this case, the decoding error (if the feedback channel is available) is bounded above by:

$$
\left|X_{1}-\hat{X}_{1}\right| \leq V_{1}
$$

where:

$$
V_{1}= \begin{cases}\frac{L_{1}}{2^{\frac{N}{M} \mathcal{R}}} & \text { if erasure does not occur } \\ L_{1} & \text { if erasure occurs }\end{cases}
$$

Then, by following this procedure, we have: At time instant $t \in\{1,2, \cdots, M-1\}$, where the feedback channel is available, $L_{t}=|A| V_{t-1}$ and:

$$
\left|X_{t}-\hat{X}_{t}\right| \leq V_{t}
$$

where: 


$$
\begin{aligned}
& V_{N j}=F_{N j} F_{N(j-1)+M-1} \cdots F_{N(j-1)+1}\left|A^{N}\right| \cdots F_{2 N} F_{N+M-1} \cdots F_{N+1}\left|A^{N}\right| F_{N} F_{M-1} \cdots F_{1}\left|A^{N}\right| V_{0} \\
& =F_{N j}|A| F_{N(j-1)+M-1}|A| \cdots F_{N(j-1)+1}|A|\left|A^{N-M}\right| \cdots F_{2 N}|A| F_{N+M-1}|A| \cdots F_{N+1}|A|\left|A^{N-M}\right| \\
& \times F_{N}|A| F_{M-1}|A| \cdots F_{1}|A|\left|A^{N-M}\right| V_{0} \\
& =2^{M j\left(\frac{1}{M j}\left(\log |A| F_{N j}+\log |A| F_{N(j-1)+M-1}+\cdots+\log |A| F_{N(j-1)+1}+\cdots+\log |A| F_{N}+\log |A| F_{M-1}+\cdots+\log |A| F_{1}\right)\right)} \\
& \times|A|^{j(N-M)} V_{0} .
\end{aligned}
$$

Box I

$$
V_{t}= \begin{cases}\frac{L_{t}}{2^{\frac{N}{M} \mathcal{R}}} & \text { if erasure does not occur } \\ L_{t} & \text { if erasure occurs }\end{cases}
$$

At time instant $t=M$, as the feedback channel is not available, a note is sent to the decoder; hence, $\hat{X}_{M}=$ $\hat{X}_{M}^{e}=A \hat{X}_{M-1}, V_{M}=L_{M}, L_{M}=|A| V_{M-1}$. Similarly, at time instant $t \in\{M+1, \cdots, N-1\}, \hat{X}_{t}=\hat{X}_{t}^{e}=$ $A^{(t-M+1)} \hat{X}_{M-1}, V_{t}=L_{t}, L_{t}=\left|A^{(t-M+1)}\right| V_{M-1}$.

Consequently, in general, at time instant $t$ :

$$
\left|X_{t}-\hat{X}_{t}\right| \leq V_{t}
$$

where:

- For $t=0$ :

$$
V_{0}=F_{0} L_{0}
$$

- For $t=N, 2 N, 3 N, \cdots$, where feedback channel is available up to the next $M-1$ time instants, we have:

$$
\begin{aligned}
V_{t}=F_{t}\left|A^{N-M+1}\right| V_{t-N+M-1}, \\
\quad t=N j, \quad j \in\{1,2,3, \cdots\},
\end{aligned}
$$

where the sequence $F_{t}\left(t \in \mathbf{N}_{+}\right)$is i.i.d. with the following common distribution:

$$
F_{t}= \begin{cases}\frac{1}{2^{\frac{N}{M} \mathcal{R}}} & P\left(F_{t}=\frac{1}{2^{\frac{N}{M} \mathcal{R}}}\right)=1-\theta \\ 1 & P\left(F_{t}=1\right)=\theta\end{cases}
$$

- For $t \in\{N j+1, \cdots, N j+M-1\}, j \in \mathbf{N}_{+}$, where the feedback channel is available, we have:

$$
V_{t}=F_{t}|A| V_{t-1}
$$

- For $t \in\{N j+M, \cdots, N j+N-1\}$, where the feedback channel is not available, we have:

$$
V_{t}=\left|A^{t-N j-M+1}\right| V_{N j+M-1} .
$$

Consequently, the equation shown in Box I is obtained. Now, from the strong law of large numbers [34], we have the following equality, almost surely:

$$
\begin{aligned}
\lim _{j \rightarrow \infty} & \frac{1}{M j}\left(\log |A| F_{N j}+\log |A| F_{N(j-1)+M-1}+\cdots\right. \\
& +\log |A| F_{N(j-1)+1}+\cdots+\log |A| F_{N} \\
& \left.+\log |A| F_{M-1}+\cdots+\log |A| F_{1}\right) \\
= & E\left[\log \left(|A| F_{1}\right)\right]=(1-\theta) \log \frac{|A|}{2^{\frac{N}{M} \mathcal{R}}}+\theta \log |A| .
\end{aligned}
$$

Consequently, as $j \rightarrow \infty$, we have:

$$
\begin{aligned}
V_{N j} & \rightarrow 2^{M j\left((1-\theta) \log \frac{|A|}{2^{\frac{N}{M} \mathcal{R}}}+\theta \log |A|\right)}|A|^{j(N-M)} V_{0} \\
& =\left(2^{M\left((1-\theta) \log \frac{|A|}{2^{\frac{N}{M} \mathcal{R}}+\theta \log |A|}\right)}|A|^{N-M}\right)^{j} V_{0} .
\end{aligned}
$$

But, as we have assumed:

$$
\inf _{\theta \in \Theta}(1-\theta) \mathcal{R}>\max \{0, \log |A|\} .
$$

For each $\theta \in \Theta$, we have:

$$
2^{M\left((1-\theta) \log \frac{|A|}{2 \frac{N}{M} \mathcal{R}}+\theta \log |A|\right)}|A|^{N-M}<1,
$$

and hence, $V_{N j}$ along with the sequence $V_{N j+1}, \cdots, V_{N j+N-1}$ converges to zero, almost surely, as $j \rightarrow \infty$. This completes the proof as $\left|X_{t}-\hat{X}_{t}\right| \leq V_{t}$.

For the vector case, the encoder encodes each element of vector $X_{t}-\hat{X}_{t}$ into $\mathcal{R}_{i}, i=\{1,2, \cdots, q\}$ bits and transmits a packet with the length of $\frac{N}{M} \sum_{i=1}^{q} \mathcal{R}_{i}$ over the compound erasure channel when the feedback channel is available.

\section{Biography}

Alireza Farhadi received $\mathrm{PhD}$ degree in Electrical 
Engineering from the University of Ottawa, Ontario, Canada, in 2007. After receiving PhD degree, Dr. Farhadi worked as Post-doctoral Fellow in the Department of Electrical Engineering of the University of Ottawa during 2008-2009 and the French National Institute for Research in Computer Science and Control (INRIA), Grenoble, France, during 2010-2011. He then worked as Research Fellow (academic level B) in the Department of Electrical Engineering of the University of Melbourne, Australia, during 2011-2013. In September 2013, he joined the Department of Electrical Engineering of Sharif University of Technology as Assistant Professor. Dr. Farhadi's areas of expertise are networked control systems, distributed optimal control, stochastic and nonlinear control, and automated irrigation networks. 